- There was a statistically significant association between mode of delivery and positive culture result, $\chi 2(1)=10.263, \mathrm{p}=$ 0.001 (Figure 2).

- Sensitivity, specificity and positive predictive value for skin swab were $36.3 \%, 86.7 \%$ and $16 \%$ respectively.

Conclusions

- Routine SSC is inefficient in predicting the pathogen responsible for sepsis among premature neonates.

- E coli was the predominant organism in the study and $37 \%$ of babies with positive SSC had E. coli sepsis (blood culture).

- Mean CRP was higher in positive skin \pm blood culture cases. However this was statistically not significant.

- There was increased risk of EONS with Vaginal delivery.

\section{PO-0556 HAND HYGIENE IN AN INDIAN NICU: SCOPE FOR IMPROVEMENT}

${ }^{1} \mathrm{R}$ Shah, ${ }^{1} \mathrm{D}$ Patel, ${ }^{1} \mathrm{~S}$ Nimbalkar, ${ }^{1} \mathrm{~K}$ Shah, ${ }^{2} \mathrm{AG}$ Phatak. ${ }^{1}$ Department of Pediatrics, Pramukhswami Medical College, Karamsad, India; ${ }^{2}$ Central Research Services, Charutar Arogya Mandal, Karamsad, India

\subsection{6/archdischild-2014-307384.1198}

Background Stringent handwashing practice is an essential component of patient care in neonatal intensive care units. We evaluated handwashing practices followed in the Neonatal Intensive Care Unit at Shree Krishna Hospital in Karamsad, India.

Methods Prospective observational study over a week in November 2013. Motion activated camera place over washbasin recorded handwashing. Six main steps i.e. step 2 to step 7 of World Health Organisation's hand hygiene technique with soap and water were used for evaluation. Handwashing was categorised as excellent if it exceeded 20 seconds and all six steps were followed, acceptable if duration exceeded 20 seconds but only 3 steps were followed. Rest was classified as unacceptable.

Results Of 1081 recordings, 775 (71.7\%) were nurses, 204 (18.9\%) were parents and $102(9.4 \%)$ were of doctors. From these, 403 (37.3\%) were excellent, 521 (48.2\%) were acceptable and $157(14.5 \%)$ were unacceptable handwash. From nurses, 82 $(10.6 \%)$, from parents $70(34.3 \%)$ and from doctors $5(4.9 \%)$ were unacceptable handwash. There were 665 (61.5\%) occurring during day time (8 am to $8 \mathrm{pm}$ ) and 416 (38.5\%) during nights. Unacceptable handwashing was more prevalent in the night as compared to daytime $(73[17.5 \%])$ vs. 84 [12.6\%], $\mathrm{p}=0.025)$. Twelve people washed their face after washing their hands in the night shift.

Conclusion The study indicates scope for improvement with emphasis on night shifts and parents. Innovative interventions may be required for parents. Empowerment of all stakeholders with reemphasis with constructive feedback may be considered along with repeat cross sectional studies to improve quality.

\section{PO-0557 NEURO-IMAGING IN INFANTS WITH CONGENITAL CYTOMEGALOVIRUS INFECTION: RELATION WITH TIME OF ONSET OF INFECTION DURING PREGNANCY}

${ }^{1} \mathrm{~N}$ Oosterom, ${ }^{1} \mathrm{~J}$ Nijman, ${ }^{1} \mathrm{~J}$ Gunkel, ${ }^{2} \mathrm{TFW}$ Wolfs, ${ }^{1} \mathrm{~F}$ Groenendaal, ${ }^{1} \mathrm{MA}$ Verboon-Maciolek, ${ }^{1} \mathrm{LS}$ de Vries. ${ }^{1}$ Department of Neonatology, Wilhelmina Children's Hospital University Medical Center Utrecht, Utrecht, Netherlands, ${ }^{2}$ Department of Pediatrics, Wilhelmina Children's Hospital University Medical Center Utrecht, Utrecht, Netherlands
Background Cytomegalovirus infection early in pregnancy results in major disabilities, including cerebral palsy and sensorineural hearing loss (SNHL). Cerebral abnormalities detected using cranial ultrasound (cUS) and magnetic resonance imaging (MRI) have been related to neurological sequelae.

Objective To evaluate additional value of MRI and assess relationship between time of infection during pregnancy and outcome in infants with congenital cytomegalovirus (cCMV) infection.

Patients and methods Demographic and clinical data were collected in infants with cCMV infection (1992-2013). Time of onset of infection during pregnancy, neuro-imaging results and outcome were reviewed. Cerebral abnormalities were categorised into none, mild (lenticulostriate vasculopathy (LSV), germinolytic cyst, high signal intensity T2 weighted images) and severe (migrational disorder, ventriculomegaly, cerebellar hypoplasia). Fisher exact test was used for statistical analysis.

Results Thirty-five infants were eligible for analysis. cUS was performed in all and MRI in 19 infants. cUS was superior for diagnosing LSV $(p<0.01)$ and MRI for diagnosing migrational disorders $(\mathrm{p}<0.01)$.

In 17 infants time of onset of infection during pregnancy was ascertained. Eight of ten infants infected during first trimester had severe cerebral abnormalities and adverse sequelae, two had no or mild cerebral abnormalities and normal outcome. Two of three infants infected during second trimester had normal outcome and one developed SNHL. All four infants infected during third trimester had normal outcome.

Conclusion Infants with first trimester cCMV infection are most at risk of severe cerebral abnormalities and neurological sequelae. MRI provides additional information for presence of migrational disorders, essential for early prediction of outcome.

\section{PO-0558 ANTI-INFLUENZA IGG LEVELS IN NEWBORN INFANTS, BORN FROM MOTHERS, VACCINATED FROM INFLUENZA AT PREGNANCY}

${ }^{1} \mathrm{MP}$ Kostinov, ${ }^{2} \mathrm{AP}$ Tcherdantsev, ${ }^{1} \mathrm{D}$ Pakhomov. 'Laboratory of Vaccination and Immunotherapy of Allergic Diseases, Institute of Sera and Vaccines, Moscow, Russia; ${ }^{2}$ Cathedra of Pediatrics, Ulianovsk University, Ulianovsk, Russia

\subsection{6/archdischild-2014-307384.1200}

Background and aims Influenza vaccination with inactivated vaccines is safe for pregnant women, as for fetus. But the levels of immunity protection against influenza in newborn infants, born from mothers, vaccinated by immunoadjuvant vaccines in pregnancy are not investigate yet. We study transplacental IgG levels in newborn infants, born from mothers, vaccinated in pregnancy by subunit influenza vaccines.

Materials and methods We study 79 infants, born from mothers, vaccinated in second and third trimester of normal pregnancy. 42 of them (first group), who's mothers were vaccinated by three-valent subunit vaccine (Grippol Plus by 'Petrovax, Ltd company, Russia, contain 0,5 mcg influenza strains and $500 \mathrm{mcg}$ of Polyoxidonium, 37 (second group) - were from mothers, vaccinated by three valent subunit vaccine ('Agrippal S1' by 'Novartis Vaccines and Diagnostic', Italy) see Table 1. Criteria of Committee for Proprietary Medicinal Products by CPMP/BWP/ 214/96 protocol be used as immunological criteria. IgG-antibody levels were measured in umbilical cord blood by hemagglutination-inhibition reaction.

Results Comparative characteristic of laboratory data achieve that in newborn infants $53,1 \%, 67,6 \%$ and $62,5 \%, 57,7 \%$ were 


\begin{tabular}{|c|c|c|c|c|c|c|c|c|c|c|c|c|c|c|}
\hline \multirow{5}{*}{$\begin{array}{l}\text { Observed } \\
\text { symptom }\end{array}$} & \multirow{5}{*}{$\begin{array}{l}\text { Terms } \\
\text { observation }\end{array}$} & \multicolumn{6}{|c|}{ I group. "Grippol plus» $(\mathrm{n}=42)$} & \multicolumn{7}{|c|}{ II group. "Agrippal S1» $(n=37)$} \\
\hline & & \multicolumn{6}{|c|}{ A/California/7/2009/ } & \multicolumn{7}{|c|}{ A/California/7/2009/ } \\
\hline & & \multicolumn{2}{|l|}{ ofH1N1/v } & \multicolumn{2}{|c|}{ A/H3N2/(Victoria) } & \multicolumn{2}{|c|}{ B(Brisbane) } & \multicolumn{2}{|c|}{ H1N1/v } & \multicolumn{2}{|c|}{ A/H3N2/(Victoria) } & \multicolumn{3}{|c|}{ B(Brisbane) } \\
\hline & & Mother & Infant & Mother & Infant & Mother & Infant & Moter & Infant & Mother & Infant & Moter & Infant & \\
\hline & & & & & & & & & & 64,9 & 65,4 & & & \\
\hline & 2-3 day newborn/ & 81,1 & 75,0 & 91,9 & 90,6 & 89,2 & 84,4 & 73,0 & 73,1 & ' & ' & 97,3 & 92,3 & \\
\hline \multicolumn{2}{|l|}{ Seroprotected(\%) } & & 53,1 & & 65,6 & & 59,5 & & 50,0 & 62,2 & & & 73,1 & \\
\hline \multirow[t]{3}{*}{ (?? titer $31: 20)$} & 3 month & 80,0 & */Ñ & 82,9 & ${ }^{*} / \tilde{\mathrm{N}}$ & 88,6 & ${ }^{\star} / \tilde{\mathrm{N}}$ & 73,0 & ${ }^{*} / \tilde{N}$ & ' & 57,7 & 81,1 & $\gamma^{*}$ & \\
\hline & & & & & & & & & & 35,1 & 34,6 & 2,7 & 7,7 & \\
\hline & 2-3 day newborn/ & 18,9 & 25,0 & 8,1 & 9,4 & 10,8 & 15,6 & 27,0 & 26,9 & $"$ & " & $\cdot$ & $' / \tilde{N}$ & \\
\hline \multicolumn{2}{|l|}{ Seroprotected (\%) } & & 46,9 & 17,1 & 34,4 & & 40,6 & & 50,0 & 37,8 & & 18,9 & 26,9 & \\
\hline \multirow[t]{3}{*}{$(? ?$ titer $<1: 20)$} & 3 month & 20,0 & */Ñ & ** & $* * / \tilde{N}$ & 11,4 & 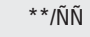 & 27,0 & $* * / \tilde{N}$ & ' & 42,3 & $* *$ & $\gamma^{* *}$ & \\
\hline & & & & & & & & & & & & 91,9 & 80,8 & \\
\hline & 2-3 day newborn/ & 64,8 & 53,1 & 78,4 & 62,5 & 75,7 & 59,4 & 67,6 & 61,5 & 62,2 & 57,7 & $\cdot$ & $\cdot$ & \\
\hline \multicolumn{2}{|l|}{ Seroprotected (\%) } & & 21,9 & & 12,5 & & 15,6 & & 34,6 & 51,4 & 30,8 & & 42,3 & \\
\hline \multirow[t]{5}{*}{ (?? titer 31:40) } & 3 month & 54,3 & $* * / \tilde{N}$ & 71,4 & **/ÑÑ & 74,3 & 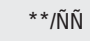 & 62,2 & $* * / \tilde{N}$ & $\cdot$ & $\gamma^{*} / \tilde{N}$ & 72,9 & $l^{* *} / \tilde{\mathrm{N}}$ & \\
\hline & & & & & & & 31,02 & \pm & & 34,82 & \pm & 85,74 & $\pm 33,64$ & \pm \\
\hline & & 38,19 & $\pm 25,78$ & $\pm 45,95$ & $\pm 31,74$ & $\pm 54,01$ & $\pm 0,19$ & 42,38 & $\pm 37,03$ & $\pm 0,68$ & & 0,28 & 0,5 & \\
\hline & 2-3 day newborn/ & 0,24 & 0,26 & 0,25 & 0,17 & 0,27 & $\tilde{N}$ & 0,39 & 0,65 & $\cdot$ & $23,78=$ & & $\tilde{N} \tilde{N}$ & \\
\hline & & & 12,59 & \pm & 14,47 & \pm & 13,50 & $\pm 27,32$ & \pm & 23,78 & $\pm 10,91$ & $\pm 47,57$ & $\pm 18,34$ & \pm \\
\hline \multicolumn{2}{|l|}{ Geometric mean } & 30,31 & $\pm 0,22$ & 30,31 & $\pm 0,18$ & 44,89 & $\pm 0,21$ & 0,38 & $20,0 \pm 0,5$ & 550,59 & 0,44 & 0,45 & 0,4 & \\
\hline ( $\log _{2} \mathrm{GMT}$ ??) & 3 month & 0,26 & $* * / \tilde{N}$ & 0,21 & $* * / \tilde{N}$ & 0,23 & $* * / \tilde{N}$ & * & * & * & $* * / \tilde{N}$ & $* *$ & $* * / \tilde{N}$ & \\
\hline
\end{tabular}

seroprotected against $\mathrm{A} / \mathrm{H} 1 \mathrm{~N} 1 / \mathrm{v}$ and $\mathrm{A} / \mathrm{H} 3 \mathrm{~N} 2 /$ accordingly, and 59,4\%, 80,8\% - against influenza B strain (p).

Conclusion When we vaccinate pregnant women against influenza - 53,1-80,8\% infants were seroprotected against vaccine strains of influenza no matter of used vaccine type.

\section{PO-0559 CANDIDA BLOODSTREAM INFECTIONS IN A NICU (2001-2012)}

${ }^{1} \mathrm{~F}$ Pulzer, ${ }^{2} \mathrm{~N}$ Lippmann, ${ }^{1} \mathrm{C}$ Gebauer, ${ }^{1} \mathrm{~A}$ Bläser, ${ }^{1} \mathrm{M}$ Knüpfer, ${ }^{1} \mathrm{U}$ Thome. ${ }^{1}$ Neonatology, University of Leipzig, Leipzig, Germany; ${ }^{2}$ Insitute of Medical Microbiology, University of Leipzig, Leipzig, Germany

\subsection{6/archdischild-2014-307384.1201}

Background and aims Recent reports indicate that the incidence of Candida ssp. bloodstream infection (BSI) is decreasing among NICU patients, and is associated with antifungal prophylaxis and the dwell time of peripherally inserted central catheters (PICCs). This study describes the epidemiology of invasive fungal infections in a single NICU.

Methods We conducted a retrospective study of invasive candidiasis occurring in 6730 infants admitted to our NICU. Positive fungal blood cultures were identified through searching our microbiology database.

Results From a total of 2637 blood cultures performed during the study period, there were $6(<0.1 \%)$ Candida spp. (Candida albicans $\mathrm{n}=5$, Candida lusitaniae $\mathrm{n}=1$ ) BSIs reported. In 3 cases premature rupture of membranes was observed, and in 2 cases maternal vaginal smears were positive for Candida ssp. All Patients with Candida albicans BSI (Gestational age 24.3 to 27.0 weeks) had PICCs, while blood cultures became positive between day 1 to 10.1 patient died on day 13. Treatment consisted in Fluconazole (67\%), Amphotericin B (33\%), and Caspofungin (17\%), partially combined. Persistent positive Candida BSIs were not reported. Systemic antifungal prophylaxis is not implemented at our NICU. During the same period 402 positive fungal cultures in 198 patients were documented from other sources.
Conclusions Even without prophylactic systemic antifungal therapy at our NICU the observed rate of Candida BSIs is low but in line with other studies. Prenatal vaginal smears might help to detect ELBW infants being at high risk for candidemia.

\section{PO-0560 DETERMINATION OF BACTERIAL AGENT IN NEONATAL SEPSIS IN NICU OF IMAM HUSSEIN HOSPITAL OF TEHRAN}

${ }^{1} \underline{Z}$ Rajabi, ${ }^{2} \mathrm{M}$ Soltandallal, ${ }^{2} \mathrm{~S}$ Yaghubi. ${ }^{1}$ Food Microbiology Research Center, Tehran University of Medical Sciences/Medical School, Tehran, Iran; ${ }^{2}$ Pathobilology, Tehran University of Medical Sciences/Medical School, Tehran, Iran

10.1136/archdischild-2014-307384.1202

Background Blood infection is one of the major causes of morbidity and mortality among the newborns especially in developing countries, at the other hand being informed about antimicrobial resistance pattern for correct treatment is necessary. So the aim is study the bacterial agent in blood infection in newborns who were admitted in NICU of Imam Hussein hospital of Tehran and survey their antibiotic resistance pattern.

Methods In this study, microorganisms of blood samples of hospitalised infant in NICU from Imam Hussein, hospital of Tehran during one year isolated, and their antimicrobial resistance pattern was studied by Kirby-Bauer test.

Results During a year study on 105 blood samples, 81(77.1\%) were gram negative and 24(22.9\%) were gram positive organisms. Klebsiella pneumonia (30.5\%), Entrobacter cloacae(21\%) and Staphylococcus epidermidis(11.4\%) were the most common gram negative and positive isolated microorganisms, respectively.

Early and Late onset septicemia in this study occurred with coagulase negative staphylococci and Klebsiella penumoniae. The most antimicrobial susceptibility in gram negative microorganisms were shown to Moxyfloxacin (88.1\%) and Ciprofloxacin $(84.2 \%)$ and in gram positive were shown to Vancomycin $(83.3 \%)$. All the gram positive microorganisms were resistance $(100 \%)$ to penicillin. 